\title{
Protée
}

\section{Du silence dans les structures : sur quelques écritures dramatiques contemporaines}

\section{Patrice Pavis}

Volume 28, numéro 2, 2000

Le silence

URI : https://id.erudit.org/iderudit/030591ar

DOI : https://doi.org/10.7202/030591ar

Aller au sommaire du numéro

Éditeur(s)

Département des arts et lettres - Université du Québec à Chicoutimi

ISSN

0300-3523 (imprimé)

1708-2307 (numérique)

Découvrir la revue

Citer cet article

Pavis, P. (2000). Du silence dans les structures : sur quelques écritures dramatiques contemporaines. Protée, 28(2), 25-34.

https://doi.org/10.7202/030591ar
Résumé de l'article

Le silence n'est pas abordé comme thème de la dramaturgie contemporaine, mais comme matière nécessaire à la constitution du texte dramatique. On le vérifie à partir d'exemples d'auteurs dramatiques contemporains français. Voir le silence n'est pas chose aisée, surtout au pays des aveugles et des sourds, mais sûrement le prix à payer pour accéder à la structure à la fois embrouillée et absente des textes contemporains. 


\section{DU SILEN CE D AN S LES STRU CTU RES: SUR Q UELQ UES ÉCRITURES DRAM ATIQ UES CONTEM PO RAIN ES}
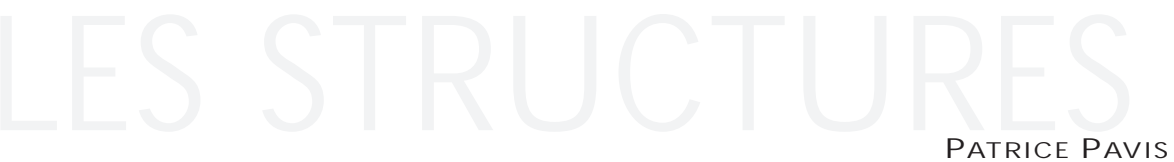

Chacun se fait en secret son idée du silence: il en a peur, elle regrette de ne pas en jouir.

Par définition indéfinissable, le silence ne se prête-t-il pas à d'infinies méditations sur l'art et la vie? Au grand désespoir du sémanticien qui ne parvient pas à le réduire à un système de catégories ou de propriétés. Le silence est devenu l'obsession d'un monde bavard et bien informé, le cri de ralliement d'un art vaincu par la difficulté de dire, le leitmotiv d'une dramaturgie du non-dit, de l'indicible ou de l'ambiguitté. Un écrivain comme Nathalie Sarraute en a fait le centre névralgique de son œuvre, non sans faire dans une pièce comme Le Silence (1967) une satire de la fascination et de l'énervement qu'il provoque chez ceux qui y sont confrontés. La plupart des pièces de ces vingt dernières années s'y réfèrent explicitement à travers les paroles des personnages ou dans leur difficulté à s'exprimer.

On pourrait donc, avec beaucoup de patience et de loisir, se livrer à une étude thématique du silence, en repérant les moments où les personnages et les didascalies évaluent ses qualités et ses fonctions. Cette étude serait interminable, car les allusions au silence sont innombrables; il est aussi souvent question du sens insaisissable des actions humaines. Nous ne nous intéresserons donc pas ici au thème du silence dans la dramaturgie, mais à sa fonction matérielle dans la constitution du texte dramatique, dans sa lecture comme dans sa représentation scénique. Le silence n'est pas une chose absente, c'est plutôt une absence chosifiée, intégrée à la "musique et la matière des mots». Plus qu'un thème, le silence est un problème technique, une manière de constituer et de lire le texte. Parler ou écrire, dans la vie et dans la littérature, c'est mettre des mots entre des blancs (des silences) et des blancs entre les mots. Sans nier ni mépriser la dimension métaphysique du silence, nous n'aurons d'yeux et d'oreilles que pour sa qualité matérielle et sa fonction pragmatique pour lire et jouer le texte dramatique.

Cette décision est confirmée par l'intuition que le silence dans l'écriture dramatique, après Tchekhov, Brecht et Beckett, n'est plus de nature psychologique, politique ou métaphysique, mais qu'il est tout simplement technique, matériel, assimilable à un silence en musique, dont l'auditeur a besoin pour entendre 
l'œuvre. Il ne s'agit plus en effet des points de suspension dont les personnages tchékhoviens faisaient un usage fréquent, ce qui donnait à leurs propos un écho toujours retentissant pour une oreille entraînée à l'analyse freudienne; pas davantage des interruptions brechtiennes où le jugement du spectateur était censé intervenir; et sûrement pas non plus des non-dits beckettiens sur le sens de Godot et de ses retards.
Le silence de l'écriture dramatique contemporaine, du moins tel que nous l'entendons ici, est autant temporel (une pause, un temps) que spatial (un blanc, un vide). C'est pourquoi on espère identifier le silence dans le processus, certes fort complexe, de la lecture du texte dramatique, en examinant à quels niveaux il est perceptible.

Le schéma de la coopération textuelle du lecteur, inspiré du modèle d'Eco dans Lector in fabula (p.88),

(A)

(B)

\begin{tabular}{|c|c|c|}
\hline $\begin{array}{l}\text { TEXTU ALITÉ } \\
\text { STYLISTIQUE : comment ça parle? } \\
\text { - M Msique et matière de mots } \\
\text { - } \quad \text { Lexique } \\
\text { - Isotopie et cohérence } \\
\text { - Intertexte et contexte } \\
\text { RHÉTO RIQUE DE LA PHRASE }\end{array}$ & $<$ & $\begin{array}{l}\text { SITU ATION D'ÉN O N CIATION } \\
\text { - Conditions de communication et d'énonciation } \\
\text { - } \text { Conscience métalinguistique } \\
\text { - } \quad \text { Rythmisation } \\
\text { - Marques de théâtralité }\end{array}$ \\
\hline \multicolumn{3}{|c|}{ TEXTE DRAMATIQ U E } \\
\hline MONDE FICTIONNEL & & MONDE DE RÉFÉREN CE ET MISE EN JEU \\
\hline $\begin{array}{l}\text { I STRU CTURES DISCURSIVES } \\
\text { THÉMATIQ UE ET SUJET : de quoi ça parle? } \\
\text { (thèmes, leitmotivs, topoï) } \\
\text { INTRIGUE: comment ça raconte? } \\
\text { RHÉTHORIQUE DES THÈMES }\end{array}$ & $>$ & $\begin{array}{l}\text { - Qu'est-ce que ça peut vouloir me dire? } \\
\text { - } \quad \text { yypothèses de lecture et de récit } \\
\text { - Qu'est-ce que la lecture (ou le jeu) fait ressortir? }\end{array}$ \\
\hline$\checkmark$ & & $\uparrow$ \\
\hline $\begin{array}{l}\text { II STRU CTU RES NARRATIVES } \\
\text { CONVENTIONS: comment ça représente? } \\
\text { FABLE : qu'est-ce que ça raconte? } \\
\text { CHRO NOTO PES (ESPACE/TEMPS) } \\
\text { DRAMATU RGIE: comment ça agit? } \\
\text { qu'est-ce que ça représente? } \\
\text { RHÉTO RIQ UE DU RÉCIT } \\
\text { («Figures textuelles») }\end{array}$ & $\longrightarrow$ & $\begin{array}{l}\text { VÉRIFICATION DES HYPOTHĖSES } \\
\text { - De quels codes disposons-nous? } \\
\text { - Qu'est-ce que ça confirme? } \\
\text { THÉO RIE DES GEN RES ET DES DISCO URS } \\
\text { - Comment l'histoire est contée? }\end{array}$ \\
\hline$\downarrow$ & & $\checkmark$ \\
\hline $\begin{array}{l}\text { III STRU CTURES ACTANTIELLES } \\
\text { ACTION : qu'est-ce que ça fait? } \\
\text { ACTANTS: qui agit? } \\
\text { RHÉTHORIQUE DES ACTANTS }\end{array}$ & $<$ & $\begin{array}{l}\text { STRUCTURES DU MONDE } \\
\text { - Accessibilité du monde possible depuis le monde } \\
\text { de référence du lecteur } \\
\text { - Quelles forces en présence? }\end{array}$ \\
\hline$\downarrow$ & & \\
\hline $\begin{array}{l}\text { IV STRU CTURES IDÉO LO GIQUES ET PSYCHANALYTIQUES } \\
\text { THĖSE : qu'est-ce que ça dit? }\end{array}$ & $\longrightarrow$ & \\
\hline INCONSCIENT DU TEXTE: qu'est-ce que ça cache? & & - Contenu latent \\
\hline & $<$ & - MeSSAge \\
\hline $\begin{array}{l}\text { LIEUX X'INDÉTERMINATION } \\
\text { ATM OSPHËRE }\end{array}$ & & - Effet produit sur le lecteur \\
\hline RHÉTHO RIQUE DU DISCOURS SOCIAL ET DE L'INCONSCIENT & & - Q uelle légitimation et interpellation du lecteur? \\
\hline
\end{tabular}


distingue ce qui est visible en surface ( $\mathrm{A}$ et $\mathrm{B}$ ) et ce qu'il faut actualiser comme contenu accessible à des niveaux plus ou moins profonds (de I à IV).

Il y a du silence à tous les étages! On ne s'en étonnera pas, puisque les structures, aux quatre niveaux, ne disent pas et ne montrent pas tout ce qu'elles représentent.

La thématique (I) par exemple ne se laisse pas toujours saisir et formuler: les thèmes y sont souvent méconnaissables ou très emmêlés. De quoi parle Pour un oui, pour un non de Sarraute? On y voit deux amis se quereller pour des vétilles stylistiques et intonatives: tout est dans la fraction de seconde, jugée trop longue, entre "c'est bien" et «ça». L'intonation révèle, supposément, mépris ou simple constatation. Ce que ça raconte n'est pas davantage résumable en une fable (II) et une dramaturgie clairement tracées. Les actants (III) sont interchangeables, ils ne représentent pas des intérêts ou des perspectives déterminés. Quant à dire ce que la pièce sous-entend, on n'en est pas beaucoup plus sûr que les personnages confrontés aux subtilités des silences...

À chacun des quatre niveaux, la structure reste ainsi très silencieuse, ce qui prouve le peu d'intérêt à les interroger pour analyser cette pièce. Comme elle n'a pas une profondeur cachée où son sens serait enfermé, il faut chercher ailleurs, tout simplement aux niveaux de surface, en $\mathrm{A}$ et $\mathrm{B}$, autant dans la «musique et la matière" que dans sa «rythmisation" et ses "marques de théâtralité». C'est du reste une caractéristique fréquente de cette écriture contemporaine: on n'y trouve rien en profondeur: fable, action, thèse ne se laissent pas clairement reconstituer. En revanche, le foisonnement stylistique de l'écriture, la manifestation ouverte de la surface textuelle sont une invite à rester en surface afin d'en décrire les mécanismes textuels. Or cette surface ne se laisse toucher et observer que si l'on admet que le texte, dans son écriture comme dans sa réception, part du silence. Le silence, le vide, le blanc sont premiers; ils génèrent la parole, le sens, la forme, comme ce qui est conquis sur le rien. Le silence de cette écriture est structural, c'est là toute la différence avec la dramaturgie classique (Racine) ou moderne (Tchékhov) dont le silence est thématique et psychologique. Dans une pièce de Tchékhov, par exemple, le texte «sécrète, «exsude» des zones de silence à l'intérieur du bruit, du trop-plein, de la parole. Les «lieux d'indétermination" (IV A) y sont des poches de résistance que combleront l'analyse et le commentaire, des galeries souterraines dans le plein du texte et du sens, des "manques" que l'inconscient du texte et du lecteur viendront combler. Dans l'écriture contemporaine pour la scène, au contraire, le silence est structural; il absorbe tout le texte, il est là avant le texte, et l'écriture doit lutter contre lui pour se constituer, pour aller du son au sens. Le silence structural est assimilable à du vide ou à de la matière qui sont autant de points de départ pour la création. Loin du silence psychologique, du sous-texte qui double et efface le texte à dire, loin d'un espace mimétique chargé de représenter le monde, le silence structural de l'écriture d'une Duras, Sarraute, Anne ou d'un Jouanneau fait la découverte du vide, dans la conception chinoise ou japonaise, d'un geste et d'une parole délivrés de l'expressivité d'un sens antérieur à transmettre: «[...] le vide est tout-puissant puisqu'il peut tout contenir. Dans le vide seul, le mouvement devient possible" (Okakura Kakuro, Le Livre du thé, 1906). Dans cette vision orientale, le vide, le blanc, le silence ne sont plus un manque à combler, un moment à passer ou un espace à remplir, mais une matière, un support pour l'écriture et le mouvement. Cette conception orientale semble s'appliquer à la manière dont le théâtre contemporain s'empare du silence pour sortir du néant. Pourtant le silence reste encore l'envers de la parole, un signifiant en instance de production de signifié, un message à communiquer, un thème et une situation fréquente dans les œuvres dramatiques d'aujourd'hui. Le silence est alors lié au sens, à l'expression, au soustexte. Il sert à nuancer, à contredire, à relativiser le sens; il est lié à une situation plutôt réaliste: un visiteur venu consulter le père de la psychanalyse et qui refuse de s'identifier (de décliner son identité) (Le Visiteur de E.-E. Schmitt); une femme juive, dont le mari est mort en déportation et qui n'a pas la force de parler de sa disparition (L'Atelier de J.-C. Grumberg); des adolescents qui n'arrivent pas à nommer les raisons de 
leur malaise (Une Envie de tuer sur le bout de la langue de $\mathrm{X}$. Durringer). Ces trois cas de figure démontrent l'usage mimétique et thématique du silence:

\section{L'inconnu}

J'ai appelé. Il n'y eut que le silence. Les carreaux devinrent plats. Ils se taisaient [...]

Et ma voix montait, montait au premier, au second, retentissait entre les murs vides où il n'y avait nulle oreille pour l'entendre. Freud

Et ma voix montait, montait... Et l'écho ne m'en revenait que pour mieux faire entendre le silence. (Le Visiteur, p. 22-23)

Cette scène raconte le moment où Freud enfant a pour la première fois vécu sa séparation d'avec le monde, un monde qui ne lui parle plus et qui ne répond pas à ses cris d'angoisse. Cet épisode du silence du monde sera une expérience fondatrice pour Freud qui cherche en vain dans son étrange Visiteur une réponse à ses questions existentielles. Freud, toutefois - et c'est là le paradoxe -, est capable d'analyser parfaitement les raisons de ce silence, qu'il s'agisse du monde de l'enfant, du croyant ou de l'angoissé, il imagine au moins une explication au silence. Son discours est maîtrisé, il s'exprime par des phrases claires et brèves, séparées par des points marquant la séparation, mais aussi l'enchaînement logique des actions et des souvenirs. Les quelques silences - qui sont davantage des pauses ou des ralentissements que des interruptions de la pensée contribuent à la clarification de la narration, à son côté inéluctable et fatal. Peu d'endroits où l'auditeur risquerait d'être induit à des significations aberrantes: la rythmisation est sous contrôle, tout au plus révélatrice d'une légère et inhabituelle hésitation du psychanalyste face à lui-même.

Dans L'Atelier le silence imprègne également tous les personnages: silence gêné des non-juifs et impossibilité pour les juifs de relater leur histoire personnelle.

MARIE: C'est votre mari qui aime pas ça [danser]?

SIMONE: (après un léger temps) Il n'est pas là, il est déporté.

Bref silence (p.137)
Comme dans Le Visiteur, L'Atelier fait coïncider le silence comme thème général et la manière silencieuse de faire échanger des silences par les personnages, de construire une série de tableaux réalistes sans que jamais l'enjeu dramaturgique ne soit expressément évoqué. Le silence est du non-dicible, ce que chacun sait (la mort des déportés), mais que personne n'ose dire, par pudeur et pour ne pas donner une réponse définitive aux questions en suspens. Seules les didascalies révèlent les hésitations en les nommant du point de vue de leur brièveté (léger temps, bref silence). L'indication de temps est une didascalie à l'intention des acteurs, pour signaler le malaise. Le silence reste intérieur à la fiction et à l'interprétation psychologique des personnages.

Il en va de même dans une autre écriture mimétique du réel, celle de Xavier Durringer dans Une Envie de tuer sur le bout de la langue:

$$
\begin{array}{ll}
\text { POUPON: } & \text { Rou! } \\
\text { ROU: } & \text { Quoi? } \\
\text { POUPON: } & \text { Rien... } \\
\text { ROU: } & \text { Si, dis-moi? } \\
\text { POUPON: } & \text { [...] j'ai une envie de tuer au bout de la } \\
& \text { langue... (p. 118) }
\end{array}
$$

Les silences, marqués par les points de suspension, sont très brefs, ils ont à peine la possibilité de se laisser théâtraliser, tant la situation est tendue et le débit rapide. Durringer ne les utilise que pour suggérer les affects et enchaîner actions et réactions. Ils sont tellement programmés et prévisibles qu'ils ne sauraient participer activement à la constitution du matériau et de sa masse amorphe, ouverte à la voix du lecteur.

De cette ouverture du texte par le silence, cette manière de le faire résonner dans l'éther de la phrase et de la scène, on prendra à présent trois exemples, chargés d'illustrer ce que nous avons nommé le «silence structural». Ces exemples seront choisis en fonction de la rythmisation qui leur est imposée - ou du moins fortement suggérée - par une ponctuation qui est une véritable "mise en scène» de la phrase.

Dans Éclats, de Catherine Anne, on a de fait l'impression de ne recevoir que des éclats d'objets 
pulvérisés et coupants, une mosaïque qui n'aurait pas encore été assemblée, parfois des éclats chatoyants et des reflets réunis par hasard.

$\begin{array}{ll}\text { AlEX: } & \text { La mayonnaise tombe } \\ \text { LAJOS: } & \text { La mayonnaise mienne } \\ \text { AlEX: } & \text { La mayonnaise tienne oui } \\ & \text { Elle ne tient pas } \\ & \text { Pas du tout } \\ & \text { Elle dégringole elle s'effondre } \\ & \text { Fonce (p.82) }\end{array}$

L'absence de ponctuation, la disposition en vers libres sont autant d'unités de souffle et de vecteurs énergétiques clairement tracés pour les comédiens. La langue théâtrale est gestuelle et les gestes vocaux, rythmiques et physiques sont ici prescrits par la syntaxe et sa disposition typographique. L'allusion à une faute de français et le jeu de mots sur tienne qui en résulte n'est pas simplement la preuve de l'habileté linguistique du personnage, elle révèle un travail de Catherine Anne sur le signifiant, une tentative pour transformer le langage en musique. En concentrant les échanges, en réduisant le langage à quelques mots ou sonorités, l'auteur s'approche du silence ou d'une polysémie propre à la poésie. Il joue sur les échos, les sonorités (effondre/fonce), les altérations, les contaminations entre les signifiés ou entre les signifiants. Sa "déponctuation " est respiratoire en ce qu'elle insuffle une vie nouvelle au langage et au monde: la communication interpersonnelle, en particulier entre les deux amies Marthe et Camille, passe par des échanges à demi-mot, dans un langage très condensé, dans une "mayonnaise» qui, elle, tient bien. Les silences dans le texte ne sont plus des sousentendus et des impressions subjectives, ce sont des «trous» qui reconstituent le texte (la mayonnaise), qui le concentrent et parfois le dénaturent, qui trouvent une autre énergie pour les échanges, comme s'il s'agissait d'inventer, grâce aux jeux du signifiant, une autre manière de communiquer.

La place et la dimension des silences contribuent à travailler de l'intérieur la matière verbale, à en modifier la composition, à en échanger le rythme et donc à constituer une écriture spécifique. Le silence n'est donc pas toujours audible/visible et matérialisé dans des points de suspension, ou une pause. Il est parfois comme intégré à l'écriture, en particulier au jeu sur le signifiant. Dans Toujours l'orage d'E. Cormann, les dialogues d'un rêve sont restitués tels quels:

NATHAN: Je les démêle, maman. Il faut tout le temps les

démêler, tu comprends.

VOIX DE FEMME: De quoi je me mêle?

NATHAN: Je l'aime, mais m'aime-telle? (p.73)

Aucune pause marquée dans la transcription/ représentation de ce rêve. Il y a plutôt une fureur de dire qui accélère le rythme, fait immerger des termes aux signifiants proches, mais aux signifiés distincts: démêle/mêle/m'aime, la série étant précédée par la forme enfantine de mère: maman. Les hésitations et les ralentissements de l'inconscient se trouvent éliminés et comprimés dans une chaîne ininterrompue et dense de signifiants. À l'image de la fable de la pièce qui, une fois lancée, ne connaît aucun arrêt et qui progresse de manière dramatique vers l'issue finale: la reconnaissance, pour le vieil acteur, d'une culpabilité et d'une fuite, et pour le jeune metteur en scène, de l'impossibilité d'échapper lui aussi à la culpabilité collective. Tous deux découvrent peu à peu ce qu'ils ont toujours réprimé et réduit au silence, Toujours l'orage fera rage en eux. De la même manière, la pièce de Cormann est bâtie comme un cauchemar à la logique implacable, mais dont on ignore jusqu'au bout le sens profond.

Le silence, ainsi donc, n'est pas toujours nettement audible, marqué par une longue pause, indiqué par une didascalie, signalé par le personnage. Souvent, c'est à l'auditeur d'entendre les ruptures entre les fragments, de mettre en mémoire la suspension d'un énoncé pour être capable de la connecter avec une nouvelle énonciation. Telle est la technique de déliaison des répliques, inventée par Tchékhov et systématisée aujourd'hui par Vinaver:

$M^{\text {me }}$ AUZANNEAU: Ça me rappelle l'orage quand la petite est partie et qu'on a su seulement après qu'elle faisait l'infirmière à l'hôpital. 
XAVIER: Vous avez de mauvais rapports avec elle?

SOPHIE: On dirait un roman qui commence.

M. Auzanneau: Et la pute. (Portrait d'une femme, p. 507)

Chacun parle sans apparemment se soucier des autres: les brefs silences qui suivent chaque réplique font apparaître d'autant plus fortement le changement de thème et produisent un effet de cacophonie. Vinaver ne touche pas au signifiant des mots, il se contente de disjoindre les fragments, de répartir un même discours sur plusieurs répliques, d'organiser les échos. Le fin travail de marqueterie déplace les césures et instaure de nouveaux silences, particulièrement éloquents. L'auditeur met par exemple ce discours en mémoire "et le connecte avec l'occurrence soudaine». Cette accusation éclaire ironiquement la question bien innocente de Xavier, l'ami de Sophie, question sans destinataire précis: prêter l'oreille, connecter les fragments, ne pas craindre d'avoir, identifier et remplir les espaces de silence, telles sont les principales tâches de l'auditeur-lecteur. La difficulté n'est donc pas de repérer les silences, car il n'y en a pas et les répliques défilent à toute allure. Elle est de faire tenir les silences entre les échos sans perdre le fil des diverses conversations. Parfois, comme dans cet extrait, la déliaison est extrême, à d'autres moments, notamment lors des duos entre Sophie et Xavier (p.505-507), les échos et les silences semblent comme expulsés et les locuteurs se répondent, en utilisant des stichomythies.

Ces trois exemples empruntés à Anne, Cormann et Vinaver soulignent l'importance de la voix dans le texte, celle du personnage-locuteur, mais aussi celle du lecteur/spectateur qui décide de la ponctuation vocale qu'il y «entend", c'est-à-dire perçoit phonétiquement et comprend. On donnera deux exemples de cette ponctuation vocale: le découpage de la phrase et le choix de son acmé.

Le découpage dramaturgique en actes, tableaux, scènes et répliques fournit un premier cadre avec des hiatus plus ou moins marqués, assurant la rupture ou la liaison des éléments. Dans l'alexandrin, des règles limitent les possibilités de la diction. Mais c'est toujours le comédien qui par son jeu (voix + corps + texte) décide des pauses et de leur durée, fixe le schéma intonatif. Qu'on songe aux deux premiers alexandrins de Britannicus:

Quoi /// tandis que Néron s'abandonne au sommeil

Faut-il que vous veniez attendre son réveil?

La voix du comédien fera un effort physique pour prolonger le quoi, avant d'ouvrir la voie à la subordonnée temporelle, par un silence que seule sa présence est en mesure de tenir.

L'écriture contemporaine est souvent beaucoup plus libre de décider de la place des pauses; le silence est parfois un véritable couperet qui fait mal et a des conséquences violentes. Ainsi dans Éclats de C. Anne, cet échange entre la mère et la fille doit être réglé avec une précision d'horloger ou de guillotineur.

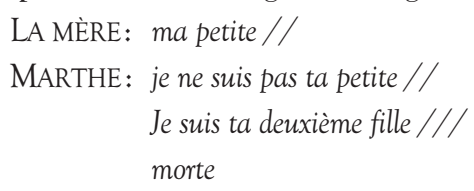

Une première pause (après petite) correspond à la typographie séparant les reprises de souffle. Une seconde vient, beaucoup plus brutalement, séparer deux membres de phrase et deux idées, avec un effet de surprise. Le cri de la mère "Marthe» est immédiat, et sa proximité renforce le parallélisme phonétique morte/Marthe qui sous-tend toute la pièce.

C'est la voix qui participe à la respiration du texte, en fonction à la fois de la ponctuation inscrite en lui (qu'elle doit ou non matérialiser clairement par des signes diactiques) et du sens que le locuteur désire lui conférer. La respiration et le sens ont partie liée: sans respiration rythmante et clarifiante, le sens n'émerge pas; sans un minimum de sens, on ne peut (faire) respirer et vivre le texte.

La voix participe à la mélodie et à l'intonation de la phrase. Là encore, l'intonation obéit à des prescriptions du texte, mais c'est le locuteur qui, en fin de compte, décide de la mélodie générale, et notamment du sommet de la phrase, ou acmé, 
moment qui termine la montée de la voix avant sa redescente. L'acmé se situe toujours à un endroit stratégique.

Soit la première phrase de Dans la solitude des champs de coton de B.-M. Koltès : "Si vous marchez dehors, à cette heure et en ce lieu, c'est que vous désirez quelque chose que vous n'avez pas, et cette chose, moi, je peux vous la fournir...» (p.9).

La phrase de Koltès continue pendant encore une page entière, mais comme il faut bien respirer, le lecteur ou l'acteur décide de l'attribution des pauses et de la gestion montée/descente de la voix en fonction des capacités respiratoires. Dans l'extrait cité, on pourrait ne réaliser qu'une seule acmé sur moi; l'on a aussi la faculté d'organiser deux acmés, la première sur lieu, la seconde sur moi. La première montée/descente restera subordonnée à la seconde. D'autant plus qu'il faut décider sur quel mot précis se fait l'acmé, sur chose ou sur moi. En accentuant chose et en coupant avant moi je peux (descendant) on ne fait de moi qu'une répétition du mot je, on insiste sur la chose et on néglige ce moi. Au contraire, en accentuant moi et en regroupant chose et moi, on insiste sur la chose, et on laisse ouverte la possibilité d'une apposition entre chose et moi: moi qui suis une chose, un objet du désir. L'ambiguïté sur l'objet du désir, la chose, est, on le voit, au cour du dispositif rhétorique de la phrase, Koltès laissant le lecteur prendre ses responsabilités respiratoires.

La question du silence n'est qu'une affaire d'écriture, et l'écriture est liée à la respiration. Le souffle est ce qui nous fait écrire et lire selon des silences, des pauses, un rythme, une matière verbale à travailler.

Où se logent les silences du texte dramatique? On a vu que l'acte de lecture est ce qui décide, en dernière analyse, de leur implantation. Dans notre schéma de la coopération textuelle, la colonne B (situation d'énonciation) est le monde de référence d'où nous interrogeons le texte à tous ces niveaux. C'est aussi la zone de silence face au sens et au bruit du texte, considéré dans sa textualité et sa fictionnalité (colonne A). La mise en jeu du texte est en effet une mise à l'écoute du texte, à partir de nos hypothèses de lecture, nos attentes, nos questions, nos sousentendus. On a déjà eu l'occasion de constater que les pièces contemporaines sont peu analysables du point de vue de leur thématique (I), de leur dramaturgie et de leur fable (II), de leur action (III) et de leur idéologie (IV), et qu'il est donc préférable de les examiner en surface dans leur textualité et leur situation d'énonciation. C'est ce qu'on vérifiera brièvement en repérant les silences et leur fonction dans quelques-unes des catégories de la textualité (A) et de la situation d'énonciation (B).

\section{MUSIQUE ET MATIÈRE DES MOTS}

Les mots sont pris, en poésie comme au théâtre, entre le son et le sens. Pour les apprécier, pour mesurer la polyphonie d'un Michel Vinaver ou d'une Catherine Anne, il faut d'abord entendre les échos, les répétitions, les allitérations, les allusions. Cette écoute musicale et rythmique n'exclut pas l'analyse discursive et linguistique, mais elle la précède. Les mêmes mots sont donc tour à tour ou simultanément signifiants et signifiés. Le même silence est à déguster tantôt comme une pause musicale avant le son, tantôt comme un effet de sens facilitant la mise en réseau sémantique des mots.

Il y a "musique et matière des mots» dès que le sens surgit non d'une intention ou d'un plan, mais d'un matériau. On a vu dans l'extrait de Toujours l'orage de Cormann (p.73) que la série démêle/même/ maman/m'aime est un pur jeu sur le signifiant m(aime). Comme pour le travail du rêve, dont il est du reste question ici, il faut partir du travail sur le signifiant. Or le silence, quoique difficilement repérable, constitue un signifiant, une matière de la musique des mots. Son absence aussi, d'ailleurs, car un discours sans aucune pause, sans ponctuation, dit d'une seule traite, fournit un matériau brut dont émerge peu à peu du sens. Dans Inventaires de Philippe Minyana, trois femmes racontent à toute vitesse leur vie pour un jeu radiophonique, interrompues seulement par le meneur de jeu. En parlant sans reprendre leur souffle, sans réfléchir, elles «sortent» des souvenirs et des 
aveux, elles «désenfouissent» des aspects oubliés de leur vie. Cette «psychanalyse du pauvre», cette "parole automatique», ce "monologue extérieur" mettent en lumière la trame de leur vie et la constitution de leur langue.

C'est là une recherche fréquente de l'écriture dramatique contemporaine: retrouver la langue dans son flux intérieur mais en extériorisant ses procédés, en les traitant comme une matière rythmique. Inventaires résume trois vies de femme en trouvant pour chacune non seulement une manière de parler caractéristique de leur milieu social, mais aussi une vitesse d'exécution, un phrasé, une rhétorique de la phrase qui sont le meilleur garant de leur identité profonde.

La langue fait sentir sa dégradation, son appauvrissement, sa déshumanisation. Ainsi dans Exécuteur 14 de Hakim, le dernier survivant de la guerre relate avec minutie le processus d'oubli qui atteint l'être humain et sa langue:

Tu perds la mémoire

Tu tombes dans le précipice où il n'y a pas de fond.

Tu tombes, et le monde passe devant tes yeux, comme un fou qui a perdu le sens. Toutes les directions sont perdues, lost pour toujours.

Tu lookes, et tu ne comprends rien à ce qui est arrivé. Tu vois seulement les yeux de petite amie ou de l'enfant. ils crient help. En silence.

Toi, tu n'as rien fait. Pas tendu la main.

Alors tu ne poses pas de questions, et no comment tu oublies. (p.35)

Un cri silencieux: cet oxymore est tout ce qui reste du monde extérieur. La langue se gangrène d'expressions anglaises toutes faites, le survivant approche de sa fin. La langue comme l'existence humaine se rabougrit, se replie sur elle-même (p.37), le silence est destructeur, surtout celui qui précède «l'explosion et la mort» (p.36).

Les conditions de communication et d'énonciation réunissent l'ensemble des informations dont nous avons besoin pour imaginer une situation dramatique à l'intérieur de laquelle les dialogues prennent leur sens. D'une manière ou d'une autre le lecteur perspicace et $a$ fortiori le metteur en scène astucieux trouvent toujours suffisamment d'indices pour reconstituer la situation d'énonciation et irriguer le texte dramatique. L'écriture contemporaine repose souvent sur une situation d'énonciation paradoxale, explicitée. Dans Via negativa de Durif, on a peu d'informations sur les personnages, leur origine ou leurs motivations, on sait seulement qu'ils se retrouvent dans un établissement psychiatrique pour expérimenter de nouveaux médicaments antidépresseurs. Leurs discours, ils en sont conscients, ne sont que des paroles (p.40):

Dans la chambre conjugale

JOSEPH: Cela fait longtemps.

BERNADETTE: Longtemps?

JOSEPH: Tu sais bien de quoi je veux parler?

BERNADETTE: N'en parlons pas.

JOSEPH: N'en parlons pas.

BERNADETTE: [...] tu es là posé à côté, plus de désir, comme on dit, mais ça ne se dit pas, ça ne se dit jamais, l'aveugle et le paralytique, on avance, côte à côte, on s'en va mais où? [...] JOSEPH: Si l'on parle, cela ne pourra être que questions réponses, ou bien là posées côte à côte paroles qui s'emmêlent par moments, ne disent rien d'autre que cela. S'échangent, tu crois? Non, ne se frôlent même pas, posées, côte à côte. Si un seul les disait, qu'est-ce que cela ferait? (p. 39-40)

Les mots, comme les deux personnages, sont ici posés l'un à côté de l'autre: il n'y a plus d'amour, plus d'échange, plus de dialectique. C'est ce que regrette Joseph et c'est ce qui rend le lecteur perplexe quand il doit interpréter ce dialogue ou la pièce tout entière. Un tel dispositif n'a pourtant rien d'inhabituel: il y a toujours du jeu entre les répliques, et cet espace vide est nécessaire à l'interprétation, car le dialogue n'est pas enchaîné à une logique causale ou temporelle des questions/réponses, il est ouvert sur de toujours possibles envolées. Le lecteur ou le metteur en scène repère les espaces de silence, en imagine d'autres, déconnecte les répliques autant qu'il les rapproche. Cette scène de Via negativa dispose les locuteurs, couple névrotico-obsessionnel, côte à côte sur un lit et 
il leur superpose des dialogues qui à la fois refusent la parole présente («n'en parlons plus», p. 39) et rejettent les futurs échanges qui ne seraient que des «questions réponses» (p.40). Cette scène est l'image même d'un dispositif d'énonciation qui pourrait être un dialogue réussi, mais qui reste une rencontre impossible. $\mathrm{La}$ communication sait qu'elle ne serait qu'une superposition de déclarations dont l'«emmêlement" est un jeu vide ne menant nulle part. Pour que la communication réussisse, il faudrait que les locuteurs acceptent de gérer, d'échanger, de partager les blancs du discours, les silences gênés, la parole commune qui sous-tend ces deux manières de parler; il faudrait que les personnages, au même titre que l'auteur et le lecteur-spectateur, acceptent la présence de silences constitutifs de toute parole.

Toutes ces conditions ressemblent fort à cette autre question de la situation d'énonciation: la conscience métalinguistique. Parfois, les pièces font allusion à leur propre fonctionnement: il s'agit souvent d'une référence à la théâtralité et aux conventions. L'allusion n'a du reste pas à être directe et destructrice de la fiction; elle est volontiers suggérée par un parallélisme des constructions, une reprise ou une réduplication. Ainsi Pour un oui, pour un non: la dispute à peine terminée, les protagonistes se lancent dans une nouvelle polémique autour du oui et du non (p.1515), le conflit portant sur la pause entre le oui et le non. Dans Art, une ironie semblable marque la pointe finale. Cette fois-ci, le blanc n'est plus scandaleux et assimilé à l'inanité de l'art moderne; il figure et représente les nuages blancs, la neige et «un homme qui traverse un espace et disparaît» (p.62). Clin d'œil de l'auteur à son lecteur, car personne n'est dupe de ces retournements ironiques.

Plus la conscience métalinguistique est sollicitée, plus l'auto-référentialité de la pièce est affirmée; plus l'insistance sur les marques de la théâtralité est grande, et plus le silence des formes se "dessine» clairement, comme si, issu des formes vides, le silence laissait voir les lignes de force, la précision géométrique. D'où cette constatation: la convention, la technique, le métalangage sont clairs et nets, car ils se dessinent sur un fond neutre, vide, silencieux, non parasité par une représentation mimétique et psychologique du monde.

D'une manière générale, on sait bien que plus la structure est abstraite et vide, plus elle est en mesure d'accueillir un texte ouvert, complexe, adaptable, poétique, à savoir éloigné du réalisme quotidien. L'écriture contemporaine est comme attirée par le vide, fascinée par le silence des formes, par la mise en vue des espaces vides. Elle rejette tout autant le nonsens de l'absurde, le non-dit du quotidien et du néonaturalisme, le non-dicible du langage, que le mysticisme du "silence éternel de ces espaces infinis» qui effrayait tant Pascal (Pensées, III, 206).

Le silence - Pascal l'avait bien vu - est pris entre l'éternel et l'infini, entre le temps et l'espace. D'où la double équation suivante:

(1) Silence + temps $=$ noir sémantique, plein de sens.

(2) Silence + espace $=$ blanc sémantique, vide du son.

- (1) En prenant le temps d'écouter un silence, on finit par lui attribuer un sens compact et précis: le silence se traduit en un sens très tangible.

- (2) En «étalant» un silence, en l'associant à une étendue, on le dilue, on en fait un support pour un sens à venir, on en reste au vide de sons non sémantisés et verbalisés.

- (1) Dans le premier cas, on fait parler le silence pour obtenir la parole; par exemple en imaginant le sous-texte, notamment aux endroits marqués comme des pauses ou des temps: travail du théâtre psychologique.

- (2) Dans le second cas, on fait taire la parole pour obtenir le silence; on réduit le langage à l'essentiel, comme pour suggérer des espaces de recueillement et de silence: travail de la poésie, et de toute écriture qui part du silence (M. Duras, N. Sarraute, C. Anne, E. Jelinek, Y. Réza, beaucoup de femmes, apparemment).

Le plus difficile n'est pas, contrairement aux apparences, de faire parler le silence, c'est plutôt de faire taire la parole. Car faire parler le silence n'exige 
qu'un peu d'imagination et l'on sait que les gens de théâtre et les piliers de bar n'en sont pas dépourvus. En revanche faire taire la parole exige que l'auditeur ou le lecteur fasse abstraction de ce qu'il entend ou voit, pour percevoir les blancs, les ruptures, les silences dans une parole ou une musique apparemment pleine: voyance de la poésie et de l'écriture dramatique d'aujourd'hui.

Il y a toutefois un danger à transformer ce silence, cette voix arrêtée, ce langage saisi dans l'instant, en un espace vide et blanc, en une structure invisible supportant l'ensemble de l'œuvre. Car cet espace, cette structure risque de se figer, d'échapper aux processus d'écoulement temporel, de devenir une forme géométrique et statique aussi raide qu'un carré sémiotique à la Greimas.

Or le silence n'est pas un état permanent, il est toujours précédé ou suivi du bruit (celui de la langue, de la musique, du sens). Il importe de savoir quand se fait le silence et quand il se défait.

La structure de l'œuvre dramatique, en d'autres termes, est fluctuante, et sa fluctuation dépend aussi (mais pas seulement) du regard et de l'écoute du lecteur ou du spectateur. Le regard est fuyant et l'écoute est flottante, ce qui nous oblige à revoir et réécouter sans cesse la frontière entre le silence et la parole. À tout moment de la lecture du texte dramatique contemporain, on doit être en mesure de modifier la rhétorique du texte (à ses cinq niveaux: voir notre schéma), notamment en vertu des interactions induites par le parcours entre les cases.

Voir le silence n'est pas chose aisée, surtout au pays des aveugles et des sourds, mais c'est sûrement le prix à payer pour accéder à la structure à la fois embrouillée et absente des textes contemporains. Apprendre à les délier, à les délire sans délirer, tel est le programme pour un lecteur trop longtemps obsédé par l'idée de faire le plein de sens. Avec le vide du son, le silence des structures, ce lecteur se retrouve dans un univers de formes silencieuses où les choses se compliquent pour lui, à force de devenir simples et ouvertes. Décidément le silence fait peur, peur d'en jouir, pour lui comme pour elle.

\section{U VRES DRAMATIQ UES CITÉES}

ANNE, C. [1989]: Éclats, Paris, Actes Sud-Papiers. Cormann, E. [1997]: Toujours l'orage, Paris, Minuit. DurIF, E. [1996]: Via negativa, Arles, Actes Sud.

DURRINGER, X. [1990]: Une Envie de tuer sur le bout de le langue, Paris, Actes Sud-Papiers, 1990.

Grumberg, J.-C. [1989]: L'Atelier, Paris, Actes Sud, 1990.

HakiM, A. [1990]: Exécuteur 14, Paris, Théâtre Ouvert.

KOLTĖs, B.-M. [1986]: Dans la solitude des champs de coton, Paris, Minuit.

MinYANA, P. [1987]: Inventaires, Paris, Éd. théâtrales, 1993.

ReZA, Y. [1994]: Art, Paris, Actes Sud-Papiers.

SARraute, N. [1967], Le Silence, dans CEuvres complètes, Paris, Gallimard, 1996;

[1982]: Pour un oui, pour un non, dans Euvres complètes, Paris, Gallimard, 1996.

SchMITT, E.-E. [1994]: Le Visiteur, Paris, Actes Sud-Papiers. VINAVER, M. [1984]: Portrait d'une femme, Théâtre complet, vol. 2, Arles, Actes Sud, 1986. 